\title{
Synthesis and Application of a Novel Polyamide Charring Agent for Halogen-Free Flame Retardant Polypropylene
}

\author{
Jie Liu, Jiayou Xu, Kaidan Li, and Yongheng Chen \\ School of Chemistry \& Chemical Engineering, Guangzhou University, Guangzhou 510006, China \\ Correspondence should be addressed to Jiayou Xu; xujiayou516@sohu.com
}

Received 5 June 2013; Revised 4 September 2013; Accepted 7 September 2013

Academic Editor: Jisheng Ma

Copyright (C) 2013 Jie Liu et al. This is an open access article distributed under the Creative Commons Attribution License, which permits unrestricted use, distribution, and reproduction in any medium, provided the original work is properly cited.

\begin{abstract}
A novel charring agent, poly(p-ethylene terephthalamide) (PETA), for halogen-free flame retardant polypropylene was synthesized by using p-phthaloyl chloride (TPC) and ethylenediamine through solution polycondensation at low temperature, and the effects of PETA on flame retardance of polypropylene (PP)/IFR systems were studied. The experimental results showed that PETA could considerably enhance the fire retardant performance as proved by evidence of the increase of limiting oxygen index (LOI) values, the results of UL-94 tests, and cone calorimeter tests (CCT). Fourier transform infrared spectroscopy (FTIR) and scanning electron microscope (SEM) demonstrated that an appropriate amount of PETA could react with PP/IFR system to form cross-link network; a more compact char layer could be formed which was responsible for the improved thermal and flame retardant properties of PP/IFR systems. However, the superfluous amount of PETA would play the negative role.
\end{abstract}

\section{Introduction}

Polypropylene (PP) is widely used in many fields, due to its easy processing property, low density, excellent mechanical properties, good performance to cost ratio, and so on. However, the use of PP in electric and electronic, building, or transport aspects is strongly limited because of their easy flammability with low LOI [1].

Thus, flame retardant PP is urgently demanded. In all plastic flame retardant technologies, the halogen-free intumescent flame retardants are promising and effective to be used in PP [2-4], due to their characteristics of low smoke, low toxicity, and no harmful gas produced during burning. Intumescent flame retardant (IFR) system is usually composed of three components: acid catalyst, carbon source, and the gas former [5]. As their name describes, intumescent flame retardants rise up in response to heat, they create a protective carbon foam under fire conditions. This class of flame retardants is a strictly condensed phase in its activity, and either provides its own carbon char or uses the polymer as a carbon char source [6]. The conventional IFRs are the phosphorus-nitrogen containing compounds; they cause a cross-linked and a thermally stable form of swollen multicellular char. Generally, the acid catalyst and gas former are ammonium polyphosphate (APP) [7] or phosphate and melamine, respectively. The choice of carbon source usually focuses on the polyol and its modified products, for example, pentaerythritol (PER) [8] or 1-oxo-4-hydroxymethyl-2,6,7trioxa-1-phosphabicyclo[2.2.2] octane (PEPA) [9]. Much of them are low molecular weight compounds; they have low thermal stability [10] and show the poor cross-linkage with the others two components. Therefore, good heat-resistant and excellent cross-linkage compounds are favorable to the carbon source.

There are already some reports for improving the thermal stability of carbon sources [11-13]; the main method is to find a compound that has excellent and good thermal stability and great cross-linking performance at the different periods of combustion. Therefore, polyamide (PA) attracts the interest of the researchers [14-17] for it has excellent char-forming capability because of the high carbon content. What is more, the thermal decomposition mechanism of amide bond is complex $[18,19]$; it is likely to generate triazine, isocyanate, or carbodiimide, which are cross-linking compounds.

However, the melting points of aliphatic PA are low which makes it easy to melt and hard to compatible with polymer materials [20] and suffer mechanical loss during the process 
TABLE 1: LOI and UL-94 results of flame retardant PP composites.

\begin{tabular}{lccccc}
\hline Samples ID & Samples & LOI & UL-94 & Dripping & Ignite the absorbent cotton \\
\hline 1 & $70 \%$ PP/27\% IFR & Y & 29 & Yes & No \\
2 & $70 \%$ PP/27\% IFR/1.5\% PETA & 31.8 & V- 0 & No & No \\
3 & $70 \%$ PP/27\% IFR/3\% PETA & 32.1 & V- 0 & No & No \\
4 & $70 \%$ PP/27\% IFR/4.5\% PETA & 32.3 & V- 0 & No & No \\
5
\end{tabular}

${ }^{*}$ The IFR here refers to the FR at 2.1.

of machining. So, we think about investigating a novel charring agent, which possesses high melting point, does not melt in the process of blending with APP, and exhibits good compatibility with most polymer materials. That is one of the most significant fields in developing the halogen-free intumescent flame retardant system.

In this paper, a novel charring agent, an oligomeric pethylene terephthalamide (PETA) was synthesized, and its thermal stability and flame retardancy in the PP-IFR system were investigated by thermogravimetric analysis (TGA), LOI test, UL-94 test, cone calorimetry test, and SEM. The aim of this study is to investigate the synergistic effects of PETA on the thermal decomposition, fire behavior, and flame retardation mechanism of PEPA/IFR/PP composite materials.

\section{Experiment}

2.1. Materials. P-Phthaloyl chloride, N-methyl kelopyrrolidide (NMP) and ethylene diamine anhydrous were obtained from Tianjin Zhiyuan Reagent Co., Ltd., China, and were used without further purification. The calcium hydroxide and calcium chloride anhydrous were purchased from Tianjin Damao Reagent Factory, China. The flame retardant (IFR) was provided by Fo Shan Magic Plastics, Fo Shan, China, which is a mixture of compounds containing phosphorus, nitrogen, and carbon.

2.2. Synthesis of PETA. Poly(p-ethylene terephthalamide) (PETA) was synthesized by using terephthaloyl chloride (TPC) and ethylenediamine (EDA) through solution polymerization at low temperature [21]. $12.4 \mathrm{~g} \mathrm{CaCl}_{2}, 6.0 \mathrm{~g} \mathrm{EDA}$, and $400 \mathrm{~mL}$ of NMP were put into a $500 \mathrm{~mL}$ three-neck flask, equipped with a mechanical stirrer. The mixture was heated to $60^{\circ} \mathrm{C}$. After the suspension was dissolved, the solution was cooled to $0^{\circ} \mathrm{C}$ under ice-water bath. Then, $20.3 \mathrm{~g}$ TPC power was dropped into the flask four times, each for $5.08 \mathrm{~g}$ with $20 \mathrm{~min}$ interval. The reaction was allowed to continue for 2 hours under $90^{\circ} \mathrm{C}$ after TPC was dropped completely. The precipitate was washed with distilled water for several times, until there was no white precipitate in the filtrate when dropwised silver nitrate. Then it was dried to constant weight at $80^{\circ} \mathrm{C}$ in vacuum oven. The yellow solid powder was obtained, with the melting point of $270^{\circ} \mathrm{C}$ and $60 \%$ yield.

2.3. Preparation of IFR Composites. The IFR composites were prepared via melt blending at $180^{\circ} \mathrm{C}$ by mixing pure PP, IFR, and PETA in Thermohaake rheomixer with a rotor speed of
$60 \mathrm{rpm}$, and the mixing time was 8 minutes for each sample. The flame retardant formulations were presented in Table 1. The samples prepared were transferred to a mold preheated for $5 \mathrm{~min}$ at $180^{\circ} \mathrm{C}$ and pressed at $10 \mathrm{MPa}$ followed by cooling to room temperature while maintaining the same pressure for $5 \mathrm{~min}$; the samples' sheets $\left(150 \times 150 \times 3 \mathrm{~mm}^{3}\right)$ obtained were stored for further tests (see Scheme 1).

2.4. Measurements. The PETA and the residual carbon samples calcined in different temperatures through the muffle furnace were characterized by FT-IR. The measurements were performed on a Vector-22 FT-IR spectrometer. Samples were mixed with $\mathrm{KBr}$ powder and pressed into tablets.

LOI values were determined using a HC-2 oxygen index meter with a sheet $130 \times 6.5 \times 3 \mathrm{~mm}^{3}$ ASTM D 2863-97. Vertical burning tests were carried out on $127 \times 12.7 \times$ $3.2 \mathrm{~mm}^{3}$ sheets according to ASTM D 3801. Cone calorimeter tests were performed using FTT cone calorimeter according to ISO $5660-1$ at a heat flux of $50 \mathrm{Kw} / \mathrm{m}^{2}$ with a sheet $150 \times$ $150 \times 3 \mathrm{~mm}^{3}$.

Thermogravimetric analysis (TGA) was performed on a TA SDTQ600 thermal analyzer at a heating rate of $20^{\circ} \mathrm{C} / \mathrm{min}$ under air condition at temperatures ranging from 25 to $700^{\circ} \mathrm{C}$.

SEM observed on a JEOL JSM-5900LV was used to investigate the surface of char residues of the PEPA/IFR/PP systems after the cone calorimeter test. SEM graphs of the char residues were recorded after gold coating surface treatment.

\section{Results and Discussion}

3.1. Structure and Thermal Stability of PETA. As shown in Figure 1, the absorption peaks at $3306 \mathrm{~cm}^{-1}$ and $1635 \mathrm{~cm}^{-1}$ correspond to the stretching vibration of $\mathrm{N}-\mathrm{H}$ and $\mathrm{C}=\mathrm{O}$ of $-\mathrm{CO}-\mathrm{NH}-$, respectively. The peak of $1292 \mathrm{~cm}^{-1}$ is assigned to the $\mathrm{C}-\mathrm{N}-\mathrm{H}$ stretching vibration. As we all know, the stretching vibration peak of C-N-H for PA-6 is $1260 \mathrm{~cm}^{-1}$, however, the peak of PETA $\left(1292 \mathrm{~cm}^{-1}\right)$ is higher than PA- 6 , which is due to the - I effect, made by the conjugation effect between benzene and carbonyl, and is lower than the $+\mathrm{C}$ effect, caused by the inductive effect between $\mathrm{N}$ and carbonyl. Additionally, the peaks of $3066 \mathrm{~cm}^{-1}, 1540 \mathrm{~cm}^{-1}$, and $1492 \mathrm{~cm}^{-1}$ are attributed to the characteristic absorption peaks of benzene ring, and the peaks at $2936 \mathrm{~cm}^{-1}$ and $2883 \mathrm{~cm}^{-1}$ are assigned to the stretching vibration of $-\mathrm{CH}_{2}-$. The typical absorption peaks of semiaromatic polyamide appeared in Figure 1, which indicates that PETA was successfully synthesized. 


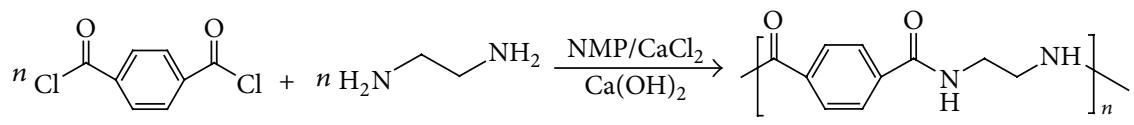

Scheme 1: The synthesis of PETA.

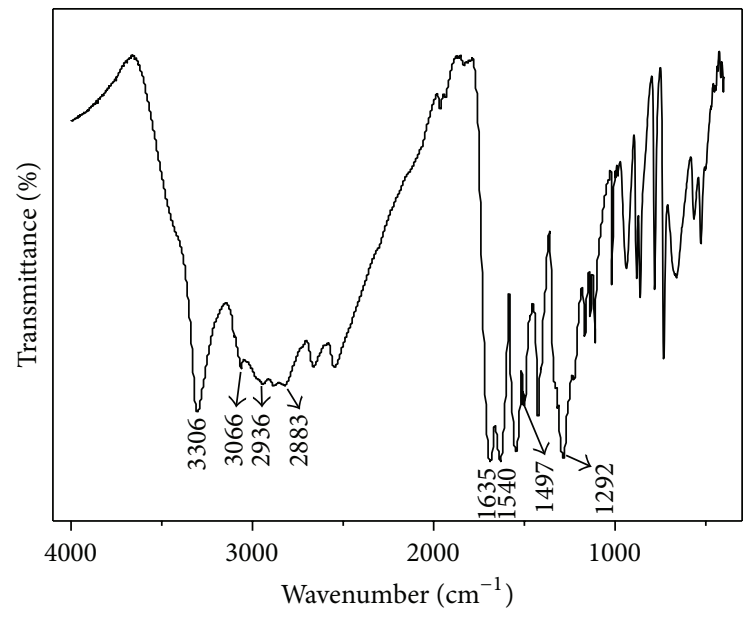

FIgURE 1: FTIR spectrum for PETA.

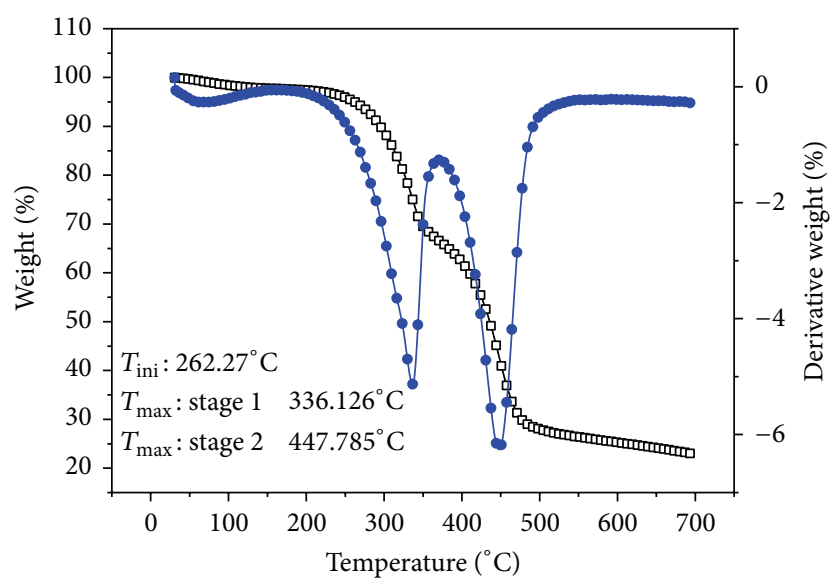

FIGURE 2: TGA and DTG curves of char-forming agent PETA.

The thermogravimetry (TG) and differential thermogravimetry analysis (DTG) curves and the related data of PETA are shown in Figure 2. The results showed that there are three peaks for PETA: the first peak around $50^{\circ} \mathrm{C}$ belongs to evaporate of water and the next two are the degradation peaks. The first degradation peak appeared in the range of $250 \sim 355^{\circ} \mathrm{C}$, and the second degradation peak is among 391 $481^{\circ} \mathrm{C}$, which is the stage of main weight loss. Between the two peaks there is a narrow space at about $35^{\circ} \mathrm{C}$ with a rather small thermal weight loss rate. It is possibly due to the degradation of low thermal stability groups in the compound, which makes the polymer fracture into many segments. At the temperature among the second degradation stage, its molecular structure collapses and the consequent thermal weight loss rate speeds up. When the temperature reaches $550^{\circ} \mathrm{C}$, the PETA decomposes completely with the residual carbon up to $23 \%$. These results prove that the semi-aromatic polyamide PETA has good heat resistance, would generate a stable compound in a relatively high temperature, and could form considerable percentages of residual carbon.

3.2. Flame Retardancy of PP Composites. LOI and UL-94 tests parameters are very important for evaluating the flame retardancy of a polymeric material. As shown in Table 1, the LOI values increase to different extents with an addition of PETA in the range of $1.5-6 \%$ levels. When the content of PETA increases to $4.5 \mathrm{wt} \%$, the LOI value reaches the maximum of $32.3 \%$. However, the LOI value begins to decrease slightly with further increase of the amount of PETA. The UL-94 has the same tendency after the addition of PETA, with the UL94 rising up to V-0 from V-1. It suggests that it may have an optimum amount of PETA, and the carbonization function is weakened when there is not enough or excess addition. We conjecture that the higher addition may weaken the crosslink of the residue. Further work is in this paper to understand the interaction of PETA with the PP-FR system.

3.3. Thermal Stability of the Flame Retardant PP Systems. TGA and DTG curves for samples 1,3 , and 5 are given in Figure 3, and the thermal degradation behaviors of the samples are experienced in two steps. The main peak of thermal degradation in the first step is about $365^{\circ} \mathrm{C}$, the weight loss rate of samples 3 and 5 were lower than sample 1 ; the temperature of thermal degradation of samples 3 and 5 in second step was larger than that of 1 , which indicates that PETA could increase the thermal stability of the PP/IFR composite during combustion. The flame retarding effect of retardant on PP is related to amount of residue char and compactness of char layer. It can be seen from TGA curves that the residual masses of PP/IFR systems are more than that of PP/IFR/PETA systems, but residual masses of sample 3 are larger than those of sample 5. Generally, the residual masses are the most important index to the IFR composites. However, it did not agree with the results of LOI and UL-94 test. It might be due to the fact that it did not form a commendable cross-link char layer, until enough PETA added into the IFR composites; as a result the compactness of char layer is not enough to prevent the oxygen and heat from reaching the underlying material. The excess addition of PETA would cause a negative effect on the compactness of char layer more work was employed to clarify the phenomenon.

3.4. Cone Calorimetric Analyses. The cone calorimeter is one of most powerful tools for evaluating the flame retardant characteristics of the polymer materials. Heat release rate (HRR), peak heat release rate (PHRR), and mass loss rate 

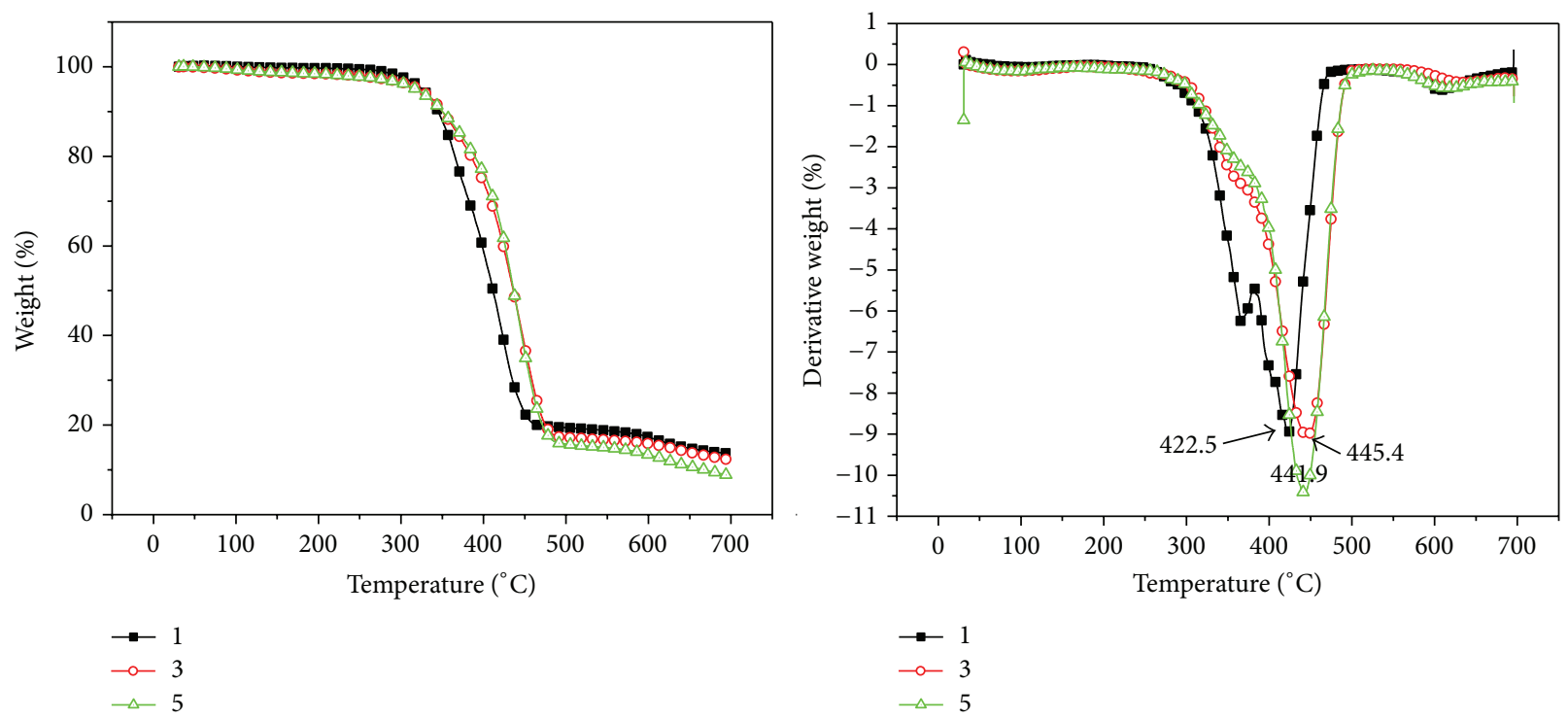

FIGURE 3: TGA and DTG curves of PP/27\%IFR, PP/27\%IFR/3\%PETA, PP/27\%IFR/6\%PETA.
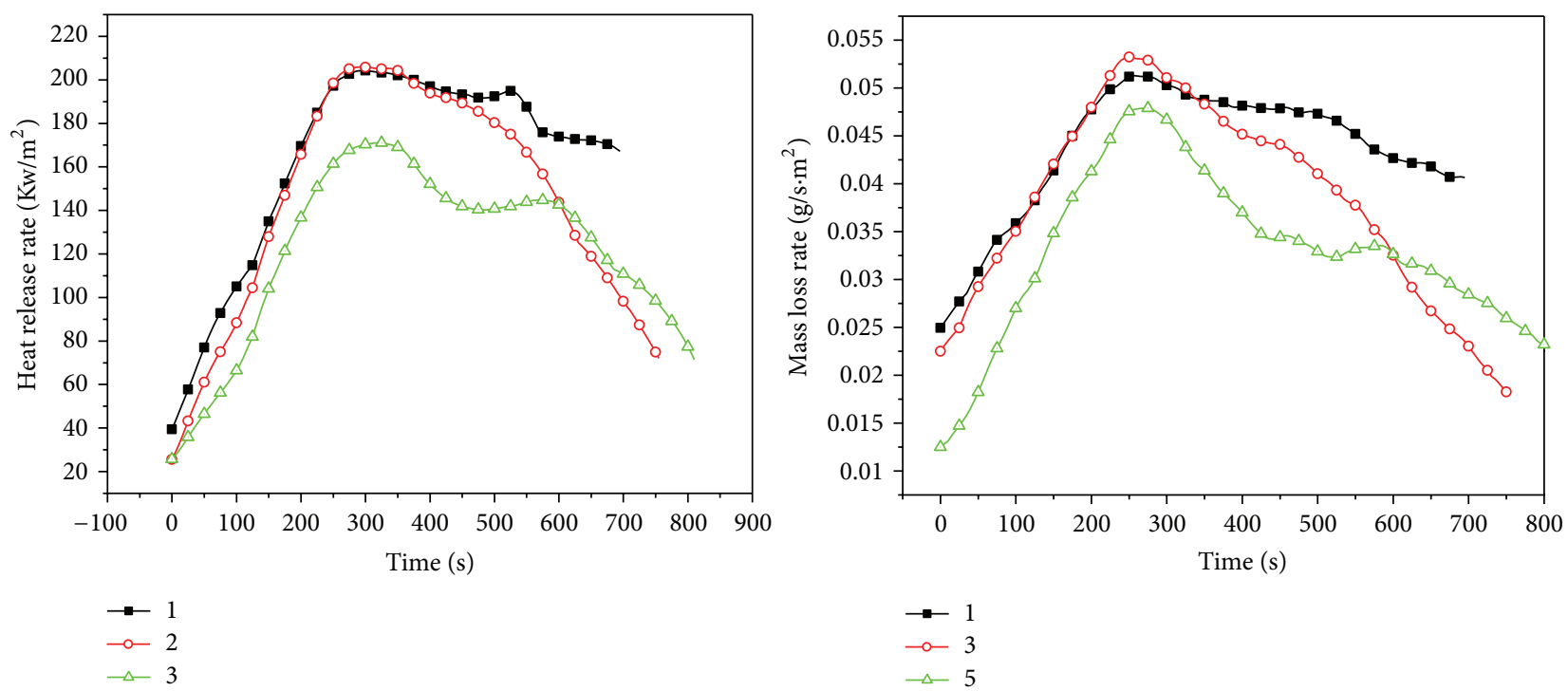

FIGURE 4: HRR and MLR curves as a function of time for PP/27\%IFR, PP/27\%IFR/3\%PETA, and PP/27\%IFR/6\%PETA.

(MLR) have been found to be the most important parameters to evaluate fire safety. The total heat release (THR), mass loss $(\mathrm{ML})$, the smoke produce rate (SPR), and total smoke produce (TSP) are also used to determine the fire safety of the polymer materials. Figure 4 is the HRR and the MLR curves of the flame retardant PP systems.

For samples 1 and 2 in Figure 4, the peaks of HRR are roughly similar, but the HRR of sample 2 is reduced to $171 \mathrm{~kW} / \mathrm{m}^{-2}$. The MLR has the same trend. The HRR of sample 1 is still in a high value after the peak temperature, however, those of samples 3 and 5 decline sharply. The decline indicates that the PP/IFR/3\%PETA and PP/IFR/6\%PETA flame retardant materials would reduce damage in the real fire disasters. It is due to the fact that with the addition of PETA, the IFR/PETA systems produce thermotolerant compounds at the peak temperatures. Additionally, there is another HRR peak for sample 5 at $590^{\circ} \mathrm{C}$, but the curve of sample 3 continues to slow down the HRR value is less than that of sample 5 when the temperature is higher than $600^{\circ} \mathrm{C}$. It confirms the idea that excess addition of PETA may cause negative effect on the thermal stability and cross-link of the residual carbon layer. The covering layer would decompose in the high temperature; it is not strong enough to isolate the nonflammable gas send out and the heat transfer into the material's surface.

The SRR and THR are also accounted as the crucial parameters to influence the personnel's escape and firemen's rescue. SRR is given in Figure 5, which reveals that the first release peak of the composites with PETA appears later than that without; the second release peaks appear as the samples 3 and 5 in order, corresponding to the HRR and MLR. Figure 5 

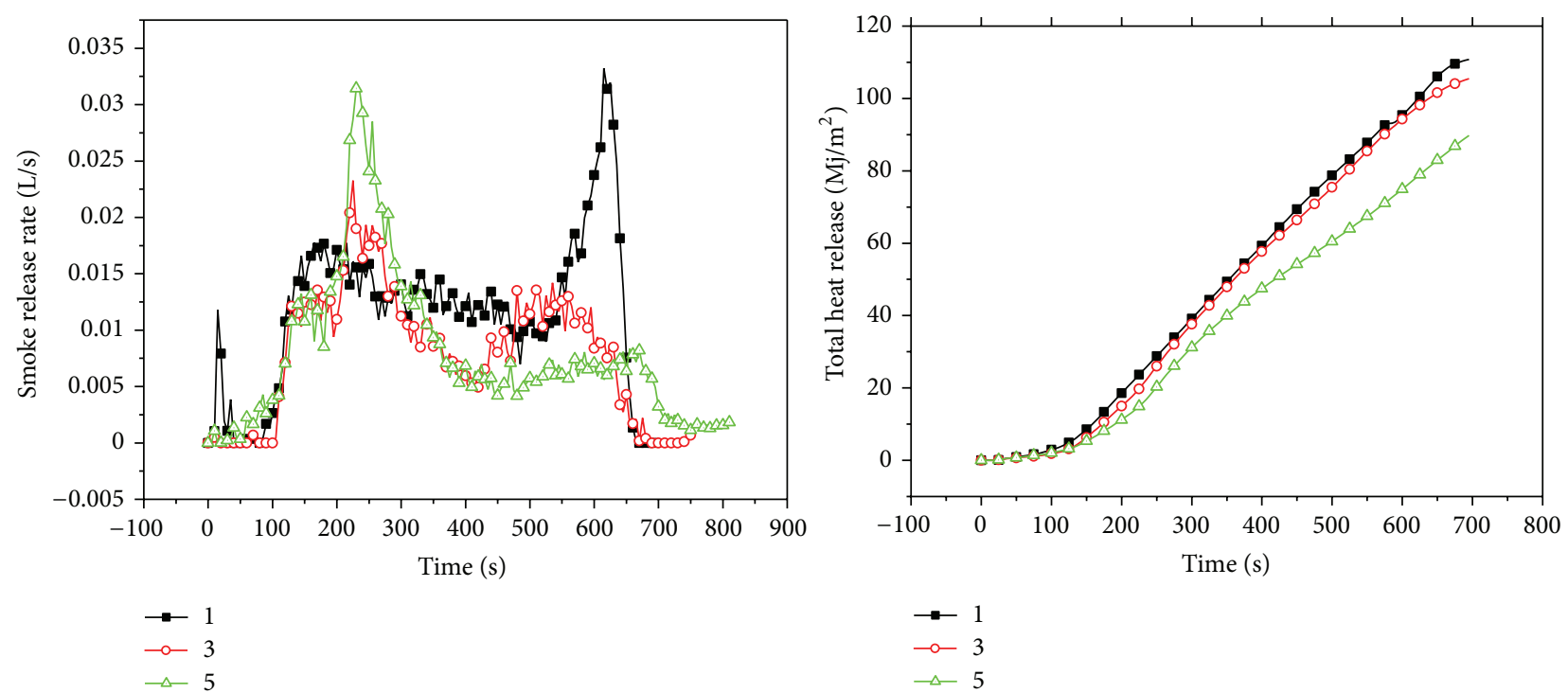

FIGURE 5: SRR and THR curves as a function of time for PP/27\%IFR, PP/27\%IFR/3\%PETA, and PP/27\%IFR/6\%PETA.
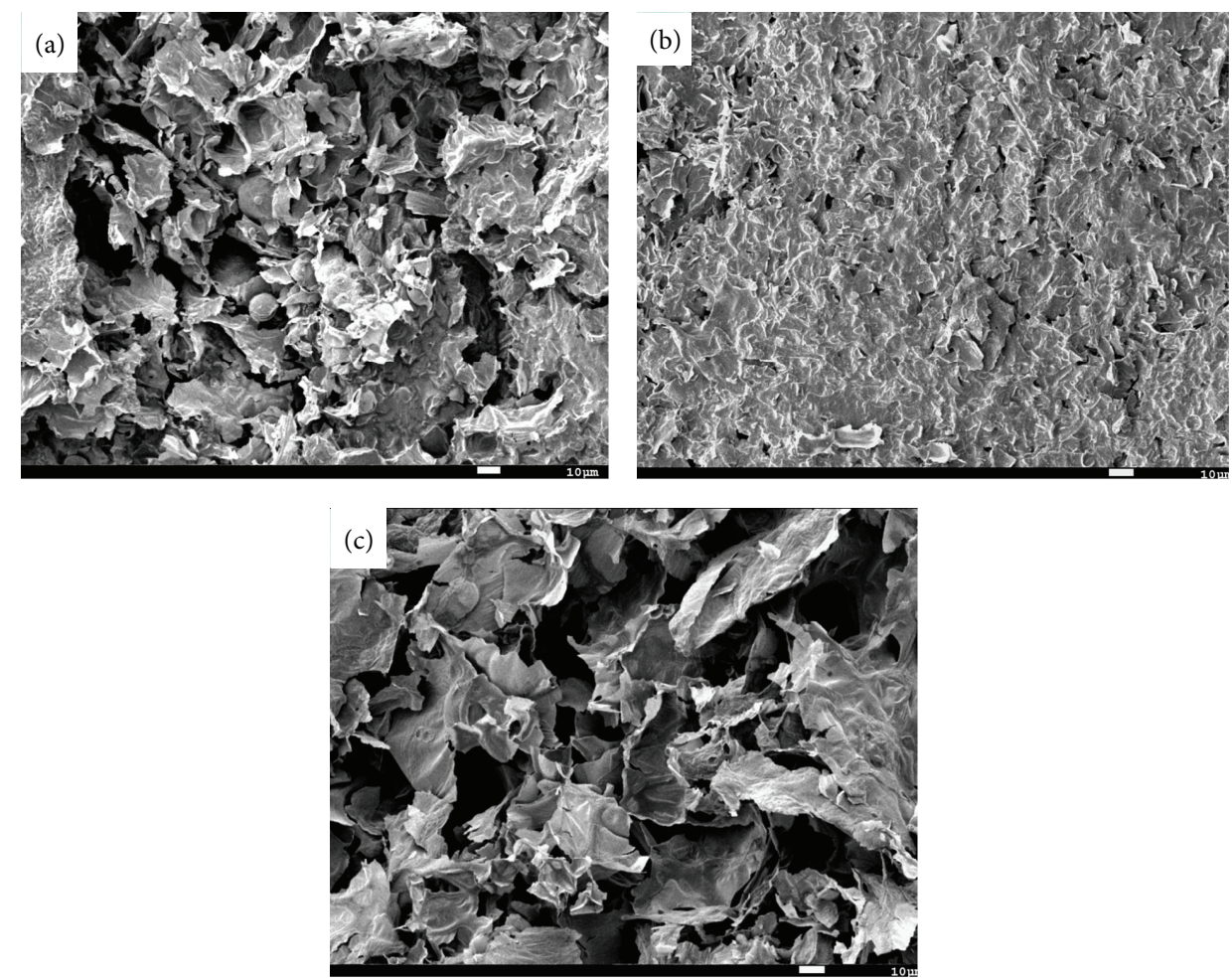

FIGURE 6: SEM images for the char residues of PP/27\%IFR systems with and without PETA: (a) PP/27\%IFR, (b) PP/27\%IFR/3\%PETA, and (c) PP/27\%IFR/6\%PETA.

shows the THR of the IFR materials. In the whole test process, the THR of sample 5 always keeps in the lowest, and the PP/IFR does the highest among them. The THR of sample 5 is the least among them; the PP/IFR sample releases the most amount of heat, which means that the PETA can reduce the quantity of releasing heat, and the more the amount of addition is, the stronger inhibitory action is. The compact char layer acts as a barrier to protect the base material from degradation and combustion, which makes THR and the related factors decrease.

3.5. Morphology of the Final Char. Figure 6 was the SEM images of the char morphology of IFR composites. The structures of samples 1 and 5 are relatively loose, and that of sample 3 is denser and more compact, which could act as an insulating barrier to prevent the oxygen and feedback of heat 


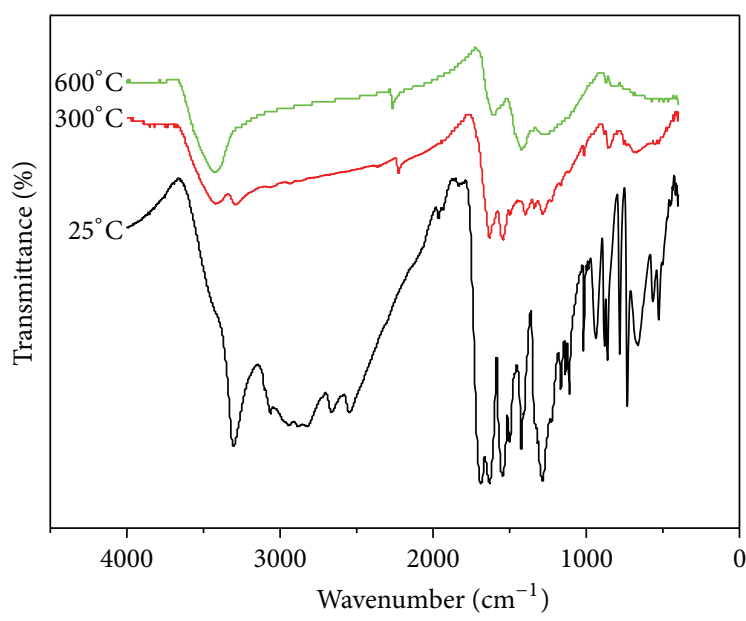

(a)

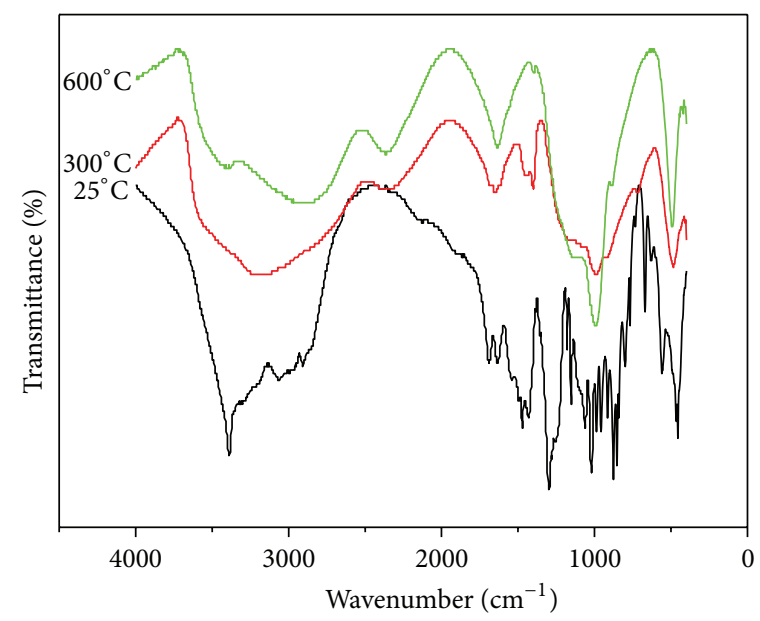

(b)

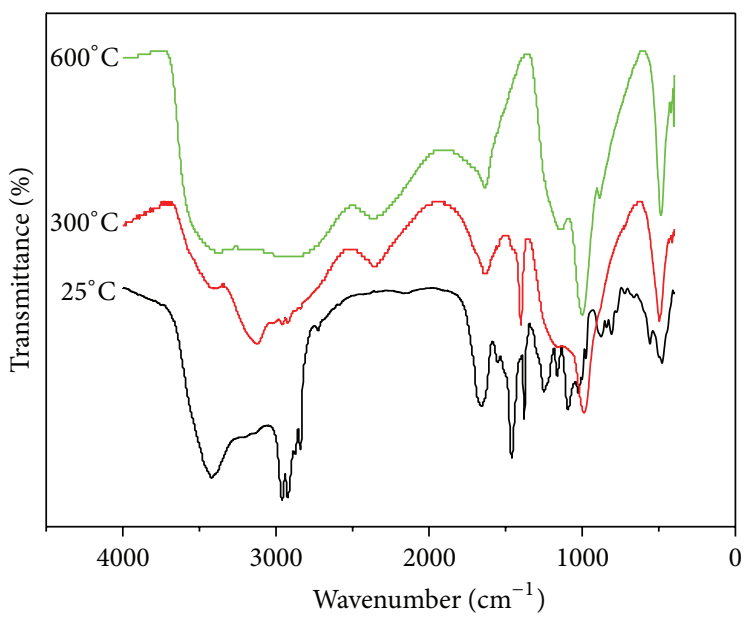

(c)

FIGURE 7: FTIR spectra at different pyrolysis temperatures of PETA (a), IFR/PETA (b), and PP/IFR/PETA (c).

from reaching the underlying material. The PETA could react with IFR system to form a cross-linked network structure char layer; consequently this makes char layer possess higher thermal stability; when the addition amount of PETA is not enough, the cross-linking ability of PETA is weak; however, the higher amount of addition might weaken the cross-link of the char residues. In a word, the appropriate amount of addition of PETA to the intumescent flame retardant PP system enhances the density and thermal stability of the residual carbon layer and improves flame retardancy of PP composites.

3.6. Flame Retardation Mechanism. Figures 7(a), 7(b), and 7 (c) show the FTIR spectra of the residues after calcined in different temperatures through the muffle furnace of PETA, IFR/PETA, and PP/IFR/PETA, respectively. At $25^{\circ} \mathrm{C}$, there are peaks at $2940 \mathrm{~cm}^{-1}$ and $2840 \mathrm{~cm}^{-1}$, which were assigned to the absorption of $-\mathrm{CH}_{2}-$. When the temperature increases to $300^{\circ} \mathrm{C}$, these peaks disappeared; it was the decomposition of $-\mathrm{CH}_{2}-$ with the increase of temperature. And the peak at $3307 \mathrm{~cm}^{-1}$, which belongs to the stretching vibration of $\mathrm{N}-\mathrm{H}$, shifts to $3418 \mathrm{~cm}^{-1}$. That was the fracture of the $-\mathrm{CH}_{2}-$ $\mathrm{NH}-$. There is a peak at $2220 \mathrm{~cm}^{-1}$ at $300^{\circ} \mathrm{C}$ in Figure 7 , which is attributed to the absorption of $-\mathrm{C} \equiv \mathrm{N}$, and the absorption peak still exists at $600^{\circ} \mathrm{C}$, which demonstrates that the $-\mathrm{NH}-\mathrm{C}(\mathrm{O})$ - in PETA could produce $-\mathrm{C} \equiv \mathrm{N}$ at high temperature, which is thermally stable at $600^{\circ} \mathrm{C}$. The degradation mechanism of PETA could be the formula (a) in Figure 8, which is similar to the degradation of PA6 [19].

PETA is added into the PP/IFR to act as the charforming agent in the flame retardant system. Consequently, an understanding of thermal degradation mechanisms of IFR/PETA and PP/IFR/PETA is very necessary. The spectra of IFR/PETA and PP/IFR/PETA are almost identical, from Figures $7(\mathrm{~b})$ and $7(\mathrm{c})$. This revealed that the degradation mechanisms of IFR/PETA with and without PP are the same.

It can be seen from Figure $7(\mathrm{~b})$ that there is a peak of $2370 \mathrm{~cm}^{-1}$ at $300^{\circ} \mathrm{C}$ and $600^{\circ} \mathrm{C}$, which does not appear at $25^{\circ} \mathrm{C}$. It might be the group of $-\mathrm{C} \equiv \mathrm{N}$ or $\mathrm{O}=\mathrm{C}=\mathrm{N}-$. The peaks of $1639 \mathrm{~cm}^{-1}$ at $300^{\circ} \mathrm{C}$ and $600^{\circ} \mathrm{C}$ are attributed to the $\mathrm{C}=\mathrm{N}$ vibration of the triazine ring or $-\mathrm{N}=\mathrm{C}=\mathrm{O}$. The possible 


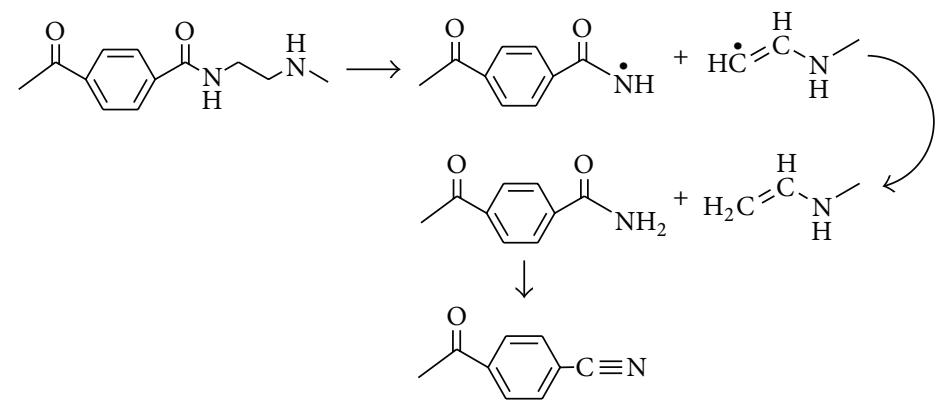

(a)

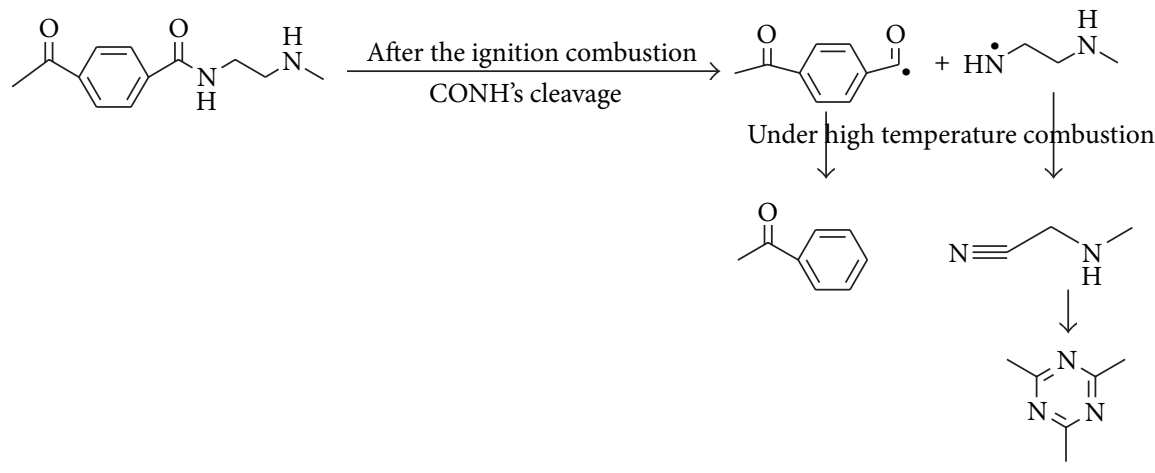

(b)

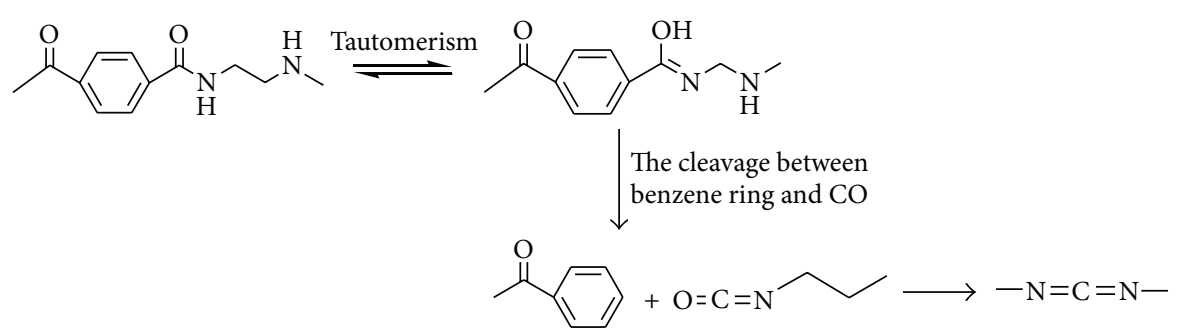

(c)

FIGURE 8: Formulas of the degradation mechanism of PETA (a) and the IFR material (b), (c).

mechanisms are shown in Figure 8, as formula (b) it forms the triazine ring eventually, and it might produce carbodiimide like formula (c). These mechanisms are side-by-side in the process of combustion. They are all different from the degradation mechanism of PETA, and what is more, heatstable compounds with cross-linking effect are created. This change demonstrates that the IFR could catalyzes the PETA to create a compound that makes the carbon layer more thermally stable and denser, which protects heat and gas from appearing between the surface and the internal at the high temperature during combustion. This carbon layer becomes carbon foam by maintaining nonflammable gases that were produced by blowing agent.

\section{Conclusions}

A novel charring agent PETA for halogen-free flame retardant polypropylene was synthesized by using TPC and EDA through solution polycondensation at low temperature. It was added into flame-retardant PP systems. The optimum addition ratio was studied, which was $3 \mathrm{wt} . \%$. The LOI to 32 and UL-94 to V-0 could be obtained when 3\% PETA was combined with $27 \mathrm{wt} \%$ IFR. The optimum amount of PETA in PP/IFR system apparently increases the thermal stability of the composite by promoting the cross-linking of carbonaceous charred layers. In addition, PETA acted as an effective flame retardant synergist to decrease inflammability and improve security by the decline of HRR, PHRR, MLR, THR, and SPR. The degradation mechanism was verified by the dynamic FTIR, which showed that PETA could react with IFR in the flame-retardant system; the IFR changed the degradation way of PETA, which generated a better thermal stability and higher cross-linked residual carbon. The morphology of the final char by SEM reflected the inference of the mechanism intuitively. The IFR/PP composite with 3 wt.\% amount of PETA was denser and more compact, which could act as an insulating barrier to prevent the oxygen and feedback of heat from reaching the underlying material. 


\section{References}

[1] P. Song, Z. Fang, L. Tong, Y. Jin, and F. Lu, "Effects of metal chelates on a novel oligomeric intumescent flame retardant system for polypropylene," Journal of Analytical and Applied Pyrolysis, vol. 82, no. 2, pp. 286-291, 2008.

[2] Q. X. Dong, M. M. Liu, and Y. F. Ding, "Synergistic effect of DOPO immobilized silica nanoparticles in the intumescent flame retarded polypropylene composites," Polymers for Advanced Technologies, vol. 24, no. 8, pp. 732-739, 2013.

[3] X. Lai, X. Zeng, H. Li, C. Yin, H. Zhang, and F. Liao, "Synergistic effect of phosphorus-containing nanosponges on intumescent flame-retardant polypropylene," Journal of Applied Polymer Science, vol. 125, no. 3, pp. 1758-1765, 2012.

[4] Y. Liu, J. Yi, and X. Cai, "The investigation of intumescent flameretarded polypropylene using poly(hexamethylene terephthalamide) as carbonization agent," Journal of Thermal Analysis and Calorimetry, vol. 107, no. 3, pp. 1191-1197, 2012.

[5] X. Chen, Y. Ding, and T. Tang, "Synergistic effect of nickel formate on the thermal and flame-retardant properties of polypropylene," Polymer International, vol. 54, no. 6, pp. 904908, 2005.

[6] A. B. Morgan and J. W. Gilman, "An overview of flame retardancy of polymeric materials: application, technology, and future directions," Fire and Materials, vol. 37, no. 4, pp. 259-279, 2013.

[7] J. Zhou, L. Yang, and X. L. Wang, "Microencapsulation of APPI and influence of microencapsulated APP-I on microstructure and flame retardancy of PP/APP-I/PER composites," Journal of Applied Polymer, vol. 129, no. 1, pp. 36-46, 2013.

[8] G. Camino, L. Costa, and L. Trossarelli, "Study of the mechanism of intumescence in fire retardant polymers-part 1 . Thermal degradation of ammonium polyphosphate-pentaerythritol mixtures," Polymer Degradation and Stability, vol. 6, no. 4, pp. 243-252, 1984.

[9] G. J. Wang, Y. Huang, and X. Hu, "Synthesis of a novel phosphorus-containing polymer and its application in amino intumescent fire resistant coating," Progress in Organic Coatings, vol. 76, no. 1, pp. 188-193, 2013.

[10] Q. L. Xiao, Y. G. Sun, and P. X. Chai, "Experimental study of the effects of thermochemical sulfate reduction on low molecular weight hydrocarbons in confined systems and its geochemical implications," Organic Geochemistry, vol. 42, no. 11, pp. 13751393, 2011.

[11] X. D. Qian, H. F. Pan, W. Y. Xing, L. Song, K. K. Y. Richard, and $\mathrm{Y}$. Hu, "Thermal properties of novel 9,10-Dihydro-9-oxa10-phosphaphenanthrene 10-Oxide-based Organic/inorganic hybrid materials prepared by Sol-Gel and UV-Curing Processes," Industrial and Engineering Chemistry Research, vol. 51, no. 1, pp. 85-94, 2012.

[12] Q. L. Tai, Y. Hu, K. K. Y. Richard, L. Song, and H. Lu, "Synthesis, structure-property relationships of polyphosphoramides with high char residues," Journal of Materials Chemistry, vol. 21, no. 18, pp. 6621-6627, 2011.

[13] M. Y. Shen, C. F. Kuan, H. C. Kuan et al., "Preparation, characterization, thermal, and flame-retardant properties of green silicon-containing epoxy/functionalized graphene nanosheets composites," Journal of Nanomaterials, vol. 2013, Article ID 747963, 10 pages, 2013.

[14] H. Vahabi, R. Sonnier, and B. Otazaghine, "Nanocomposites of polypropylene/polyamide 6 blends based on three different nanoclays: thermal stability and flame retardancy," Polimery, vol. 58, no. 5, pp. 350-360, 2013.

[15] S. H. Qin, S. J. Hu, J. Yu, Z. Luo, J. Guo, and W. Yan, "Preparation and combustion behavior of polyamide 6/polypropylene/clay nanocomposites," Journal of Macromolecular Science Part B, vol. 50, no. 12, pp. 2243-2254, 2011.

[16] F. Samyn and S. Bourbigot, "Thermal decomposition of flame retarded formulations PA6/aluminum phosphinate/melamine polyphosphate/organomodified clay: interactions between the constituents?" Polymer Degradation and Stability, vol. 97, no. 11, pp. 2217-2230, 2012.

[17] Z. L. Ma and W. Y. Zhang, "Study on intumescent flame retardant PP reinforced by nylon," China Plastics Industry, vol. 33, no. 2, pp. 58-59, 2005.

[18] W. Y. Jung and J. I. Weon, "Characterization of thermal degradation of polyamide 66 composite: relationship between lifetime prediction and activation energy," Polymer, vol. 36, no. 6, pp. 712-720, 2013.

[19] R. Li and X. Hu, "Mechanism of thermo-oxidative degradation of polyamide," Acta Polymerica Sinica, no. 2, pp. 136-141, 2000.

[20] J. S. Yi, Y. Liu, and D. D. Pan, "Synthesis, thermal degradation, and flame retardancy of a novel charring agent aliphatic-aromatic polyamide for intumescent flame retardant polypropylene," Journal of Applied Polymer Science, vol. 127, no. 2, pp. 1061-1068, 2013.

[21] D.-D. Yuan, H.-Q. Yin, X.-C. Ren, and X.-F. Cai, "Synthesis and application of a charring agent semi-aromatic polyamides," Journal of Sichuan University, vol. 44, no. 1, pp. 177-182, 2012. 

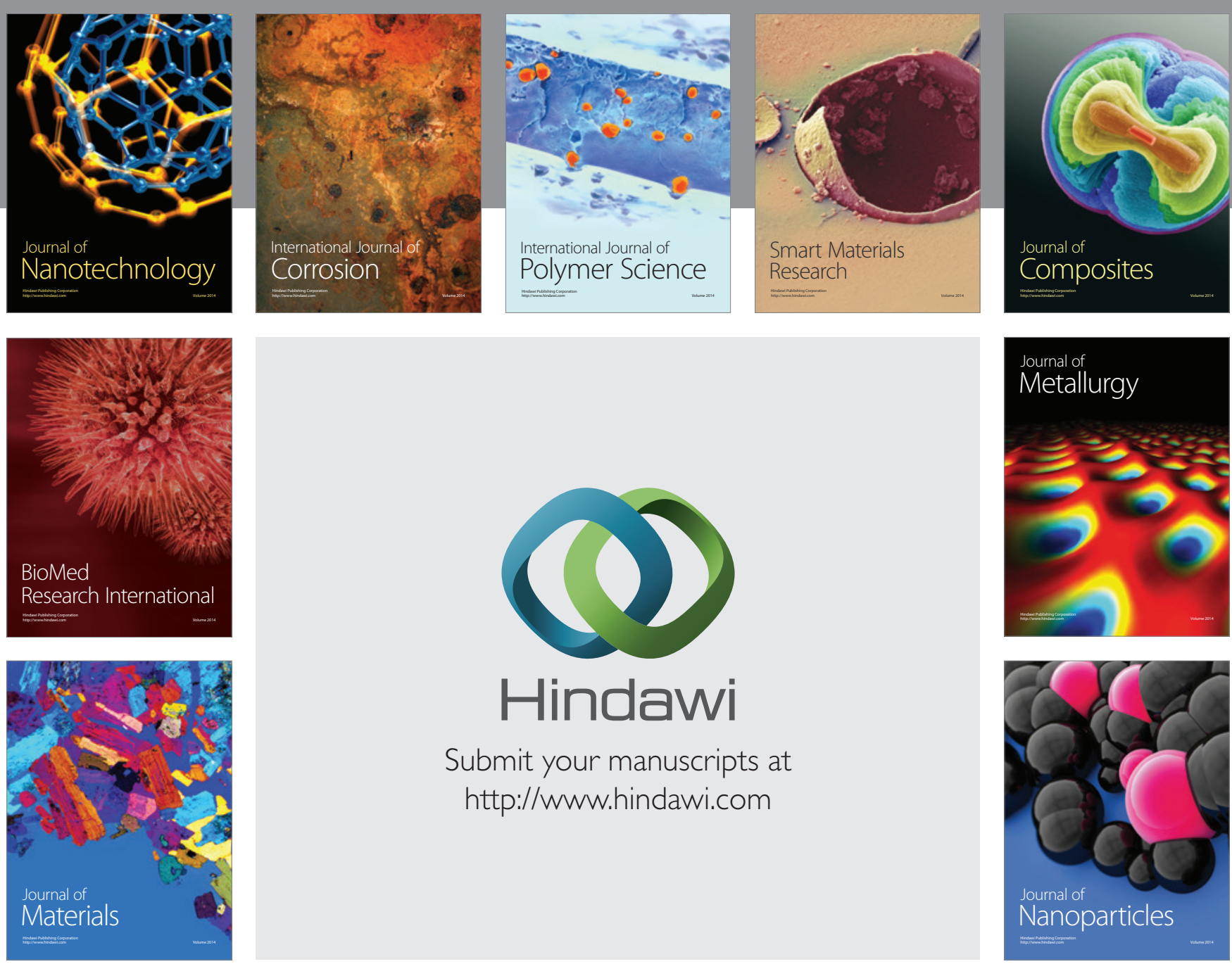

Submit your manuscripts at http://www.hindawi.com
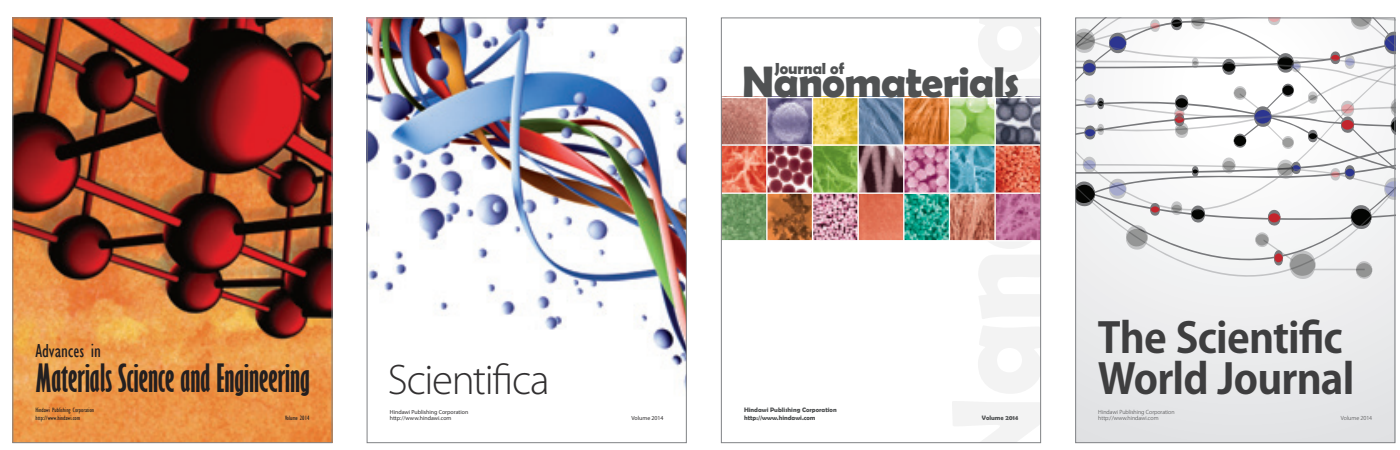

\section{The Scientific World Journal}
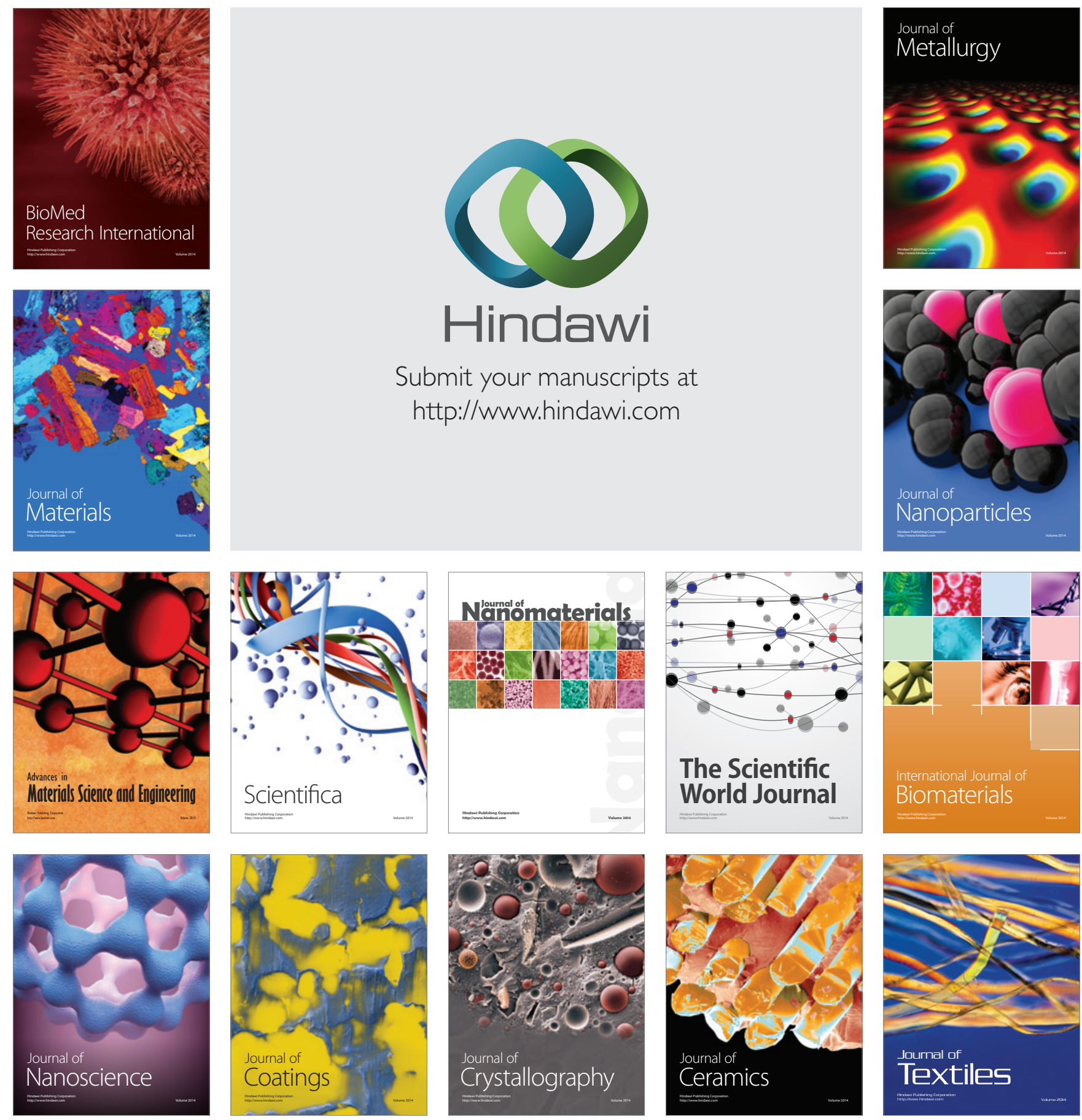